Article

\title{
On a Self-Consistency Thermodynamical Criterion for Equations of the State of Gases in Relativistic Frames
}

\author{
Gonzalo Ares de Parga ${ }^{1, *}$, Adriana Ávalos $\operatorname{Vargas}^{1}$ and Benjamín López-Carrera ${ }^{2}$ \\ ${ }^{1}$ Departamento de Física, Escuela Superior de Física y Matemáticas, Instituto Politécnico Nacional, U. \\ P. Adolfo López Mateos, Zacatenco, C. P. 07738, México D.F., Mexico \\ ${ }^{2}$ Departamento de Formación Básica, Escuela Superior de Cómputo, Instituto Politécnico Nacional, U. \\ P. Adolfo López Mateos, Zacatenco, C. P. 07738, México D.F., Mexico
}

* Author to whom correspondence should be addressed; E-Mail: gadpau @ hotmail.com; Tel.: +52-555-729-6000 ext. 55317; Fax: +52-555-729-6000 ext 55015 .

Received: 9 March 2013; in revised form: 2 April 2013 / Accepted: 2 April 2013 /

Published: 9 April 2013

\begin{abstract}
More than three decades ago, Tykodi and Hummel proposed a procedure to investigate the self-consistency thermodynamical criterion for equations of the state of gases. The main criterion used by these authors consists of requiring that an equation of state must not be inconsistent with certain thermodynamical identities from the first and second laws of thermodynamics. This paper explores the possibility of another self-consistency method based on relativistic transformations of the thermodynamical variables. It is shown that the virial coefficients have to be corrected by a relativistic factor if the equation of state is considered in a moving relativistic reference frame. Some relativistic and non-relativistic equations of state are analyzed.
\end{abstract}

Keywords: equation of state; relativistic transformation of temperature; virial coefficients

\section{Introduction}

In 1973, Tykodi and Hummel [1] analyzed the general structure of the state equations to obtain a procedure that allows the derivation of new equations of state empirically, but that would meet certain basic relations of thermodynamics. In fact, they developed a method to investigate the thermodynamical self-consistency of the equations of state for gases. Indeed, by means of the first and second laws of 
thermodynamics, they arrive at a series of equations (Equations (6) and (7) of Tykodi and Hummel [1]) that would supposedly differentiate between equations of state for gases that are physically acceptable or not. Moreover, the gas must behave as an ideal gas when the particle number, $N$ is kept fixed for a volume, $V$, approaching infinity, that is, for a dilute gas limit, i.e.,

$$
\lim _{N \text { fixed, } V \rightarrow \infty} \frac{P V}{N k T}=1
$$

They explored further consequences of Equations (6) and (7) and also derived additional constraints. However, Angulo-Brown et al. [2] have demonstrated that some conditions imposed by Tykodi and Hummel are too restrictive, and the universe of equations of state that satisfy the first and second laws of thermodynamics is broad (they proved that Equation (1) can be substituted by two constraints, $\lim _{V \rightarrow 0} V f(V)=a$ and $\lim _{V \rightarrow 0} V \xi(T, V)=b$ with $a+b=1$, where $f$ and $\xi$ have been previously defined by Tykodi and Hummel [1]). However, as noted above, many equations of state are empirically constructed and should be subject to physical rules, such as happens with the demand that for large volumes, a gas must behave as an ideal gas. However, other criteria can be used in order to verify the physical validity of an equation of state, as, for example, among others, Carathodory's (1909) proposal [3,4]. Indeed, Carathodory's assumption, "In every neighborhood of every state, $x$ there are states, $y$, that are not accessible from $x$ via quasi-static adiabatic paths, that is, paths along which $Q=O$, , permits the understanding that an adiabatic curve never crosses an isothermal curve twice, and if an equation of state predicts the contrary, it is not physically acceptable. Surprisingly, Special Relativity gives us another criterion to rule out equations of state in relativistic reference frames. Indeed, the relativistic transformation laws of thermodynamical quantities [5] allow the correction of certain equations of state when looking from a relativistic moving frame. In this work, a relativistic approach that analyzes the form of the complete equations of state is proposed, in order to correct their expression in a relativistic moving frame.

The laws of relativistic transformation of thermodynamical quantities were developed by Planck [6] and Einstein [7]. However, due to Einstein's doubts about the theory [8], new proposals appeared. Ott [9], Landsberg [10] and Rohrlich [11], among many others [12-16], developed a series of different models (see the Balescu [15] and Nakamura [17] tables). Indeed, Ott's proposal [9] represents one of the most important proposals in the field, such that Möller [18] changed his point of view about the theory. Recently, based somewhat on the Möller point of view, Requardt [19] developed a covariant proposal, which is able to show many gaps and inconsistencies in Ott's and Rohrlich's theories. Additionally, Przanowski and Tosiek [20] have newly argued that the Ott-Möller theory represents the most natural relativistic transformations of heat and absolute temperature. They based their interpretation on the recent verification of the Jüttner distribution function [21] by computer simulation [22]. However, nowadays, the covariant Israel formulation [13,14], based on van Kampen theory [12], has been recognized as the best proposal, since it comes from fundamental principles. Nevertheless, Nakamura [17] has pointed out that even if Israel's work represents a consistent covariant theory, it does not recognize that simultaneity must be taken into account at the moment of defining the temperature and the flux of heat. His analysis leads him to demonstrate that three basic formulations can be derived from the Israel proposal just by considering different choices of the decomposition of the energy-momentum into work and heat. A similar theory has been obtained by Ares de Parga 
and López-Carrera [23], where different covariant approaches of the laws of relativistic transformation quantities can be derived, coinciding with part of Nakamura's proposal [17]. In fact, Ares de Parga and López-Carrera [23] obtained the Planck-Einstein theory, with some modifications. By using this approach, a criterion to rule out equations of state, in relativistic reference frames, will be developed.

This work is organized as follows: Section 2 is devoted to briefly describing the relativistic transformation laws of thermodynamical quantities, which allow for the understanding of the invariant form of the equation of state in any reference frame. Also, in this section, a discussion about the validity of the equation of energy will be analyzed. In order to apply the relativistic criterion broadly, the complete description of a thermodynamical system is described. In Section 3, the state equation for a typical case, the van der Waals gas, is examined. In order to analyze the transformations of the virial coefficients, in Section 4, the virial expansion is studied from a relativistic moving reference frame. Following the same idea, in Section 5, the ideal Fermi and Bose gases and the blackbody are also analyzed. In Section 6, the validity of an equation of state constructed from the relativistic theory of the mean value is verified. A special section, Section 7, is devoted to study an equation of state, which fulfills all consistency relations from standard thermodynamics in the rest frame, but which would be ruled out unless the newly derived constraints from relativistic thermodynamics are used. Indeed, the criterion allows for the identification of the generalization of an existing equation of state, so that it becomes applicable in relativistically moving reference frames. Finally, some concluding remarks are presented in Section 8.

\section{Relativistic Transformations and Equations of State}

A few years ago, it was shown that the Jüttner distribution [21] in a moving system is valid through computational methods by Cubero et al. [22]; the result can be understood by considering the temperature as an invariant. This can be seen as proof that the covariant Israel theory explains the relativistic transformation laws of the thermodynamical quantities. By analyzing the tensor structure for a non-stationary irreversible thermodynamical system, Israel discussed the second law of thermodynamics for the exchange of heat and matter between bodies with arbitrary relative velocities. By using the van Kampen 4-vector temperature and defining a 4-vector flux of entropy, he arrived at the conclusion that the temperature is a Lorentz invariant. However, Nakamura [17] has shown that by using Israel formalism and noticing that there are many ways to decompose the energy-momentum into work and heat, different theories can be obtained. It is interesting to note that from the covariant theory developed by Ares de Parga and López-Carrera [5,23], based on Nakamura formalism [24], it is possible to obtain the Jüttner distribution of a moving gas when the so-called Planck-Einstein case is analyzed. In order to obtain this last result, it should be reiterated that the Planck-Einstein theory suffered a modification, principally in the transformation law of energy (the Planck-Einstein case) [5]. Although it has not been done, it is possible that by applying the Rohrlich-Gamba case [5] which represents the Ott proposal with some modification, the Jüttner distribution will be also recovered. At this point, we should mention that since Balescu [15] published his famous article about Relativistic Statistical Thermodynamics, it has been shown that many different proposals are equivalent and just differ from the Planck-Einstein theory by a kind of gauge theory adapted to Relativistic Thermodynamics. A similar 
idea has been obtained by Dunkel et al. [25], who argued that the Planck-Einstein theory is just one among several equally valid theories of Relativistic Thermodynamics. Moreover, due to the Cubero et al. result [22], it can be deduced that if the Jüttner distribution's temperature parameter is directly identified with the thermodynamic temperature, theories with Lorentz-invariant temperature are favored. However, as we mentioned before, by applying the Nakamura formalism to the Planck-Einstein case, the Jüttner distribution is recovered [5]. Finally, we can conclude that the transformation relativistic laws of thermodynamical quantities depend on the choice of the decomposition of the energy-momentum, which is related on the definition of a thermometer. This thermometer defines the reference frame where the thermodynamical quantities are measured, that is, the simultaneity. The choice of the reference frame where the thermodynamical quantities are measured is the cornerstone of the proposal done by Ares de Parga and López-Carrera [23], and this represents a similitude with the Nakamura proposal [24].

By correcting some aspects of the van Kampen theory, Ares de Parga and López-Carrera [5,23] were able to find a covariant theory of the laws of the relativistic transformations of thermodynamical quantities based on Nakamura's ideas. Let us briefly expose the theory: the main thesis consists in defining a four-vector volume in a covariant form; that is:

$$
V_{K}^{\mu}=\frac{w^{\mu} V_{o}}{w^{\lambda} u_{\lambda}}
$$

where $V_{o}, w^{\mu}$ and $u^{\mu}=(\gamma, \gamma u / c)$ represent the rest volume of the system, the time-like unit vector that defines the direction of the three-dimensional volume in the four-dimensional space and the relative four-velocity between the moving frame, $K$, and the rest frame, $K_{o}$, respectively. The values of $w^{\mu}$ and $u^{\mu}$ depend on the choice of the system where simultaneity is defined. It should be noted that for a certain choice [5,23], $w^{\mu}=(1,0)$ and $u^{\mu}=(\gamma, \gamma u / c)$, the Planck-Einstein theory is recovered with some corrections. A very important aspect of the theory is that the product of the two four-vectors, $w^{\mu}$ and $u^{\mu}$, is a characteristic quantity, which may be included in different relationships. That is, for the Planck-Einstein case:

$$
w_{\mu} u^{\mu}=\gamma
$$

the value of $\gamma$ represents the motion of the moving frame and may be included in the equations of state. The model is founded on whether the system is composed of interacting particles or not. The thermodynamical quantities must fulfill the following transformation laws, when looking at the thermodynamical system in equilibrium, from a reference frame moving with respect to the reference frame at rest of the system [5,23]:

$$
T=\gamma^{-1} T_{o}, \quad V=\gamma^{-1} V_{o}, \quad \mu=\gamma^{-1} \mu_{o}, \quad F=\gamma^{-1} F_{o} \quad \text { and } \quad \xi=\gamma^{-1} \xi_{o}
$$

where the subscript, " $O$ " refers to quantities measured in the reference system at rest, and $T, V, \mu, F$ and $\xi$ represent the temperature, the volume, the chemical potential, the Hemholtz free energy and the redefined energy, respectively. The redefined energy at rest coincides with the internal energy of the system at rest and in the moving frame, and is defined as:

$$
\xi=E-\frac{\mathbf{u}}{c} \cdot \mathbf{M}=\gamma^{-1} \xi_{o}=\gamma^{-1} E_{o}
$$

where $E, u$ and $M$ represent the internal energy, the relative motion between both frames, $\left(u^{\mu}=(\gamma, \gamma u / c)\right)$, and the total momentum of the system, respectively. It has to be noticed that 
Equation (5), which describes the way the energy has to be redefined in the Planck-Einstein case, permits one to obtain the Jüttner distribution of a moving gas [5]. As we have noticed before with some differences [23], the Ott proposal can be obtained by considering $w^{\mu}=(\gamma, \gamma u / c)$ and $u^{\mu}=(1,0)$. Also, following the formalism of this theory, it will be possible to obtain the Jüttner distribution (but, this will be the goal of a future article). Returning to the Planck-Einstein case, it should be noticed that the definition of total momentum of the system must be done in a covariant form [5,23]. On the other hand, there are invariant quantities, such as the pressure and the entropy among others:

$$
P=P_{o}, \quad S=S_{o} \quad \text { and } \quad q=\frac{1}{k \gamma T} m c^{2}=\frac{m c^{2}}{k T_{o}}=q_{o}
$$

where $m$ represents the mass of each particle in the gas.

Our purpose consists of finding a relativistic criterion that corrects or rules out the equations of state in the rest frame when they have to be expressed from a relativistic moving frame. Let us see an example.

It is very common to describe an equation of state for a gas by means of a function between the pressure, volume and temperature, that is:

$$
f\left(P, V_{o}, T_{o}\right)=0
$$

This description of the gas is incomplete, and normally, we need the equation of the energy or entropy to have the whole scenario. This point will be analyzed later in this section.

For the moment, let us stay just with the simple case described by Equation (7). Thus, it is possible to solve the pressure as a function of volume and temperature:

$$
P=P\left(V_{o}, T_{o}\right)
$$

Since the pressure is an invariant, Equation (6), the volume and temperature in the moving system can be replaced by using Equation (4), which means:

$$
P=P(\gamma V, \gamma T)
$$

We shall see later that it is not necessary to consider relativistic gases to use this method. However, in the case of an ideal Maxwell-Boltzmann gas, the relativistic equation of state coincides with the non-relativistic ideal gas [26]. In the rest frame, the equation of state is:

$$
P V_{o}=N k T_{o}
$$

By substituting the volume and the temperature in the moving frame in Equation (4), we obtain:

$$
P V=N k T
$$

That is, in this case, the same form of the state equation is recovered.

Now, it may be suspected that any equation of state must satisfy the invariance of the form. But, this first approach does not ensure such invariance for any equation of state. However, the Helmholtz free energy is a function of the volume, the temperature and the parameter, $\gamma$ which describes the motion of the system; that is [15]:

$$
F=F(V, T, \gamma)
$$


Therefore, claiming the invariance of the form of the thermodynamics quantities in a moving frame $[5,15,23,27]$, the pressure in the moving frame must be:

$$
P=P(V, T)=-\left.\frac{\partial F}{\partial V}\right|_{T}
$$

It is known how the Helmholtz free energy and the volume are transformed in Equation (4); that is:

$$
P=-\left.\frac{\gamma^{-1} \partial F_{o}}{\gamma^{-1} \partial V_{o}}\right|_{T_{o}}=P\left(V_{o}, T_{o}\right)
$$

Comparing Equations (13) and (14), we notice that the functionality of the pressure in $V$ and $T$ must be the same as the one for $V_{o}$ and $T_{o}$, due to the transformation laws in Equation (4). Therefore, we can conclude that the equation of state of any gas is an invariant in the sense that substituting the transformed thermodynamical quantities, given by Equation (4), in the equation of state in the moving frame that the equation of state in the rest frame is recovered and vice versa. This represents a relativistic criterion for any equation of state.

However, the complete description of a thermodynamical system cannot be reduced to a simple equation. On this point, Callen says [28] : "the relation that gives the entropy as a function of the extensive parameters is known as a fundamental relation. It therefore follows that if the fundamental relation of a particular system is known, all conceivable thermodynamics information about the system is ascertainable from it". That is, we must know:

$$
S=S\left(U, V, N_{1}, \ldots, N_{r}, \gamma\right)
$$

Following Callen: "the temperature, pressure and electrochemical potentials are partial derivatives of the entropy, $S$, the volume, $V$, the numbers of particles, $N_{1}, \ldots N_{r}$ and, consequently, they re also functions of $S, V, N_{1}, \ldots N_{r}, \gamma$. We must have a set of functional relationships:

$$
\begin{aligned}
T & =T\left(S, V, N_{1}, \ldots, N_{r}, \gamma\right) \\
P & =P\left(S, V, N_{1}, \ldots, N_{r}, \gamma\right) \\
\mu_{j} & =\mu_{j}\left(S, V, N_{1}, \ldots, N_{r}, \gamma\right)
\end{aligned}
$$

Such relationships, expressing intensive parameters in terms of the independent extensive parameters, are called equations of state"... "The knowledge of all the equations of state of a system is equivalent to knowledge of the fundamental equation and, consequently, is thermodynamically complete." Some combinations of Equation (16) lead to other types of equations of state, as the ideal gas equation, Equation (11). Although this kind of equation must meet the relativistic criterion, it does not possess all the information of the system. Therefore, it must be required that the fundamental relation meets the relativistic criterion. That is: the requirement that the entropy must be expressed in an invariant form represents the relativistic criterion. Indeed, the Planck-Einstein theory predicts that the entropy is an invariant, as in Equation (6). However, it will be interesting to analyze all kinds of equations of state, but the invariance of the entropy expression must be always checked, that is, the fundamental relation. Since some doubts may appear about applying this criterion to the ideal Fermi and Bose gases, a special subsection will be presented in Section 4. In this section, just the ideal gas will be analyzed. 
It should be noted that one might be tempted to use Statistical Physics, since it is known that:

$$
F_{o}=-k T_{o} \ln Z_{o}
$$

In a first approach, claiming the invariant form of Statistical Mechanics, we could use the same definition in the moving frame for the Helmholtz free energy:

$$
F=-k T \ln Z
$$

It has been demonstrated by many authors $[5,12,14-16]$ that the partition function is an invariant for systems of non-interacting particles. Therefore, considering the transformation law of the temperature in Equation (4), we can guarantee the consistency between Equations (17) and (18). In the general case of interacting particles systems, the no interaction theorem [29] does not permit us to develop a Relativistic Statistical Mechanics theory. That is, it only allows us to propose a Statistical Physics in moving frames just for non-interacting particles systems, as, for example, the ideal gas. Based on covariant arguments, a Relativistic Statistical Physics can be achieved, provided that the center of mass is well-defined. The discussion of the existence or not of a covariant center of mass is still an open problem, and we refer the reader to the works by Pryce [30], Thomas [31,32] and Curie [29]. Although, nowadays, Gill and Zachary [33] have proposed an interesting covariant definition of the center of mass, which seems to be consistent with the Lorentz group, the discussion still remains. However, Relativistic Thermodynamics does not have such a problem and a covariant relativistic theory has been developed without contradicting the no interacting problem [5]. Moreover, in spite of the no interaction theorem, some Relativistic Statistical Physics are developed by using the Mean Field Theory. Even though, the risk of not being covariant is high. Therefore, any equation of state must satisfy this relativistic criterion. That is why Equations (17) and (18) were not used to show the invariance of the equations of state, and we demonstrated it by using Redefined Relativistic Thermodynamics. It is always possible to question how the rest frame of a system is defined without a formal definition of the center of mass. The answer belongs to the theory itself, since the rest frame is defined as the frame where the temperature is higher [5]. Therefore, Relativistic Thermodynamics can be used independently if the system is composed of interacting particles or not, and the proof of the invariance of form of the equations of state is universal.

The thermodynamical description of a system, as we noticed above, is sometimes not well understood. Indeed, the equation of state does not completely describe the system. One needs to know the fundamental relation. However, it is interesting to study the equation of the energy. This equation of energy must be also taken into account for the relativistic consistency of the system. This is accomplished by knowing the heat capacities of the system. For instance, in the rest frame:

$$
C_{V o}=\left.\frac{\partial E_{o}}{\partial T_{o}}\right|_{V_{o}}
$$

By claiming the invariance of the form of the thermodynamical quantities in a moving frame $[5,15,23,27]$, the relativistic generalization of Equation (19) is:

$$
C_{V}=\left.\frac{\partial \xi}{\partial T}\right|_{V}
$$


By using the transformation laws in Equation (4), we obtain

$$
C_{V}=\left.\frac{\gamma^{-1}}{\gamma^{-1}} \frac{\partial E_{o}}{\partial T_{o}}\right|_{V_{o}}=C_{V_{o}}
$$

That is, the heat capacity at constant volume is an invariant, and it represents another relativistic criterion.

Let us show that this criterion works for some typical cases. In the case of the non-relativistic ideal gas, the experiment and the Non-Relativistic Statistical Physics tell us that for monoatomic gases, the heat capacity at constant volume is constant:

$$
C_{V o I G}=\frac{3}{2} N k
$$

and for diatomic gases:

$$
C_{V o I G D}=\frac{5}{2} N k
$$

both at low temperature. When the temperature increases, the gases are no longer perfect, and the heat capacity increases with the temperature. For ultrarelativistic monoatomic ideal gases, the heat capacity turns to [26] (high temperature):

$$
C_{V_{o} U R}=3 N k
$$

The general expression can be calculated from Statistical Mechanics, and the expression is:

$$
C_{V o}=\frac{N m c^{2}}{T_{o}}\left\{q_{o}+\frac{3}{q_{o}}-\frac{K_{1}\left(q_{o}\right)}{K_{2}\left(q_{o}\right)}\left(3+q_{o} \frac{K_{1}\left(q_{o}\right)}{K_{2}\left(q_{o}\right)}\right)\right\}
$$

where $q_{o}=\beta_{o} m c^{2}$ with $\beta_{o}=\frac{1}{k T_{o}} ; K_{1}$ and $K_{2}$ represent the Hankel functions with an imaginary argument. Those cases were exposed in order to verify that the expressions of the heat capacities satisfy the relativistic criterion. That is, it has to be questioned if the above results, Equations (22), (24) and (25), meet the relativistic criterion, even if the first two equations, Equations (22) and (24), trivially satisfy the requirement, since the calculations of the heat capacities on those cases in a moving frame by using Statistical Mechanics are quite direct. However, Equations (22) and (24) are just special cases of the general result, Equation (25). Therefore, it is just necessary to show the invariance of the general heat capacity. Nevertheless, for the general case, care has to be taken. Indeed, if we calculate $C_{V}$ by using the partition function, expressed as a function of the temperature in the moving frame [5], $T$, it is easy to see that we just need to interchange $u_{o}$ by $q=m c^{2} / k \gamma T$ (note that $q=q_{o}$, Equation (6)) leading to the same result. That is, $C_{V}=C_{V_{o}}$. Moreover, if a direct calculation of the heat capacity is done and using the invariance of the partition function [5,23], the result is:

$$
C_{V}=N k q\left\{q+\frac{3}{q}-\frac{K_{1}(q)}{K_{2}(q)}\left(3+u \frac{K_{1}(q)}{K_{2}(q)}\right)\right\}
$$

which evidently is invariant. Equations (11) and (26) do not completely describe the system. We need to know the entropy, which can be calculated just by partially deriving, with respect to the temperature, and keeping constant $V$ and $N$, the Helmholtz free energy; that is:

$$
S=N k\left[\ln \left\{4 \pi \frac{\gamma V}{N}\left(\frac{m c}{h}\right)^{3} \frac{K_{2}(q)}{q}\right\}+4+u \frac{K_{1}(q)}{K_{2}(q)}\right]
$$


It has to be noticed that $\gamma$ appears in the equation making $\gamma V$ an invariant. Even if we have demonstrated the invariance of the heat capacity for the ideal gas by using a statistical model, the main result is independent of Statistical Mechanics. Therefore, since normally the equation of the energy, that is, the heat capacities, are experimentally obtained in a rest frame, the relativistic criterion gives us the method to correct the coefficient in order to obtain an invariant result. For example, when dealing with non-perfect gases, the heat capacities will depend on the temperature, that is:

$$
C_{V_{o}}=a_{o}+b_{o} T_{o}+c_{o} T_{o}^{2}+\ldots
$$

The expression in the moving frame will just be:

$$
C_{V}=a+b T+c T^{2}+\ldots
$$

with:

$$
a=a_{o}, \quad b=\gamma b_{o}, \quad c=\gamma^{2} c_{o} \quad \ldots
$$

Something similar will also occur when a proposed equation of state or a virial expansion are analyzed. The following sections will be partially devoted to study this point. Normally, as a referee indicated to us, complete equations of state are usually expressed in the gas rest frame. Consequently, the equations may not meet our criterion at first instance. However, the technique developed in Equations (28)-(30) shows how an expression in the rest frame could be generalized to one in the moving frame. Particularly, special subsections advocated to the study of the ideal Fermi and Bose gases and the blackbody cases will be presented in Section 4.

\section{3. van der Waals Gas}

It is interesting to note that the criterion for all types of equations of state can be used whether the gas is relativistic or not. In fact, the behavior of a non-relativistic gas, that is, a low temperature gas, can be studied from a moving relativistic frame by using the relativistic criterion. Indeed, the relativistic transformation laws are valid for low temperatures, since Equation (14) is independent of the value of the temperature, and the moving frame can have a relativistic relative motion with respect to the rest frame. That is, it is always possible to consider a gas at low temperature from a reference frame in relativistic motion. Moreover, most of the cases deal with non-relativistic systems, and the equations of state are built by using adjustment methods, development of virials, etc. The method allows for analyzing whether the constants or coefficients meet the relativistic criteria. Therefore, all the results of the non-relativistic ideal gas are satisfactory. The van der Waals gas represents a similar case. Indeed, van der Waals proposed a correction to the ideal gas equation by considering certain internal effects of real gases. He obtained an equation of state that better represents a non-relativistic real gas and is expressed as follows [34]:

$$
\left[P+\left(\frac{N}{V_{o}}\right)^{2} a_{o}\right]\left[V_{o}-N b_{o}\right]=N k T_{o}
$$

where $a_{o}$ and $b_{o}$ represent the constants related to the effective radius of the molecules and $a_{o}$ is also related to the interaction of the molecules. In order to make this equation of state meet the relativistic criterion, it is clear that the constants in the moving frame must be replaced by:

$$
a=\gamma^{-2} a_{o} \quad \text { and } \quad b=\gamma^{-1} b_{o}
$$


leading to the equation of state in the moving frame:

$$
\left[P+\left(\frac{N}{V}\right)^{2} a\right][V-N b]=N k T
$$

This case demonstrates that when certain constants are proposed in an equation of state, they must be defined in a moving system, such that the invariance of the form of the equation is preserved. Therefore, it is necessary to review the meaning of both $a_{o}$ and $b_{o}$. It is well known that the van der Waals equation can be obtained from the potential of Sutherland [34] using Statistical Physics. The calculation leads us to define the constants $a_{o}$ and $b_{o}$ as:

$$
a_{o}=\frac{2 \pi}{3} r_{o}^{3} U_{o}=\frac{1}{2} v_{o} U_{o} \quad \text { and } \quad b_{o}=\frac{2 \pi}{3} r_{o}^{3}=\frac{1}{2} v_{o}
$$

where $r_{o}$ and $U_{o}$ represent the radius of the minimal potential (the radius of the modeled spheres is $r_{o} / 2$ ) and the value of the minimal potential, respectively. Since the co-volume, $b_{o}$, is the occupied volume by a molecule, $v_{o}$ it also behaves as a volume. Even if the Sutherland potential is defined as function of the radius $r_{o}$ :

$$
\Lambda_{o}=\left\{\begin{array}{cc}
\infty & r<r_{o} \\
U_{o}\left(\frac{r_{o}}{r}\right)^{6} & r \geq r_{o}
\end{array}\right\}
$$

it does not represent a potential that transforms under a relativistic rule, as, for example, the four-vector potential in Electrodynamics. However, when Statistical Mechanics is used [5], the product, $\beta \Lambda=\frac{\Lambda}{k T}$ is equal to $\frac{\Lambda_{o}}{k T_{o}}$. Therefore, $U_{o}$ must transform as an energy [5,23]. Therefore, these quantities transform in the moving frame as:

$$
v=\gamma^{-1} v_{o} \quad \text { and } \quad U=\gamma^{-1} U_{o} .
$$

By using Equation (35), it is noticed that Equations (32) and (33) satisfy the desired relativistic criterion.

\section{Virial Coefficients}

In the case of a system of interacting particles, it is known that it is not possible to define an acceptable Hamiltonian that meets the symmetries expected in relativistic physics. Indeed, as a consequence, there is no way of obtaining a canonical transformation between different reference frames. That is, the non-interaction theorem [29] prohibits the development of a consistent Relativistic Statistical Physics. Some authors deal with the problem by putting aside the Minkowski postulate (world lines), and they handle the problem through various techniques involving the fields, as for example, by using the Mean Field Theory. The latter technique allows the use of the virial expansion for relativistic or non-relativistic systems. Let us postpone the analysis of the relativistic expansion at the end of the section. At this stage, we briefly describe the virial expansion for the non-relativistic case [34]. A gas, in its rest frame, can be described by:

$$
\frac{P V_{o}}{N k T_{o}}=\sum_{l=1} a_{o l}\left[\frac{\lambda_{o}^{3}}{\frac{V_{o}}{N}}\right]^{l-1}
$$

where $a_{o l}$ and $\lambda_{o}$ represent the well-known virial coefficients and the thermic wavelength, respectively. It is clear that the left hand side of this equation is invariant, since:

$$
\frac{P V_{o}}{N k T_{o}}=\frac{P V}{N k T}
$$


Therefore, the right hand side must be invariant too. That is, the transformations of the coefficients, $a_{o l}$ and $\lambda_{o}$, must be found in order to ensure that the virial expansion meets our criterion. Since $\lambda_{o}$ is a thermal wavelength, $\lambda_{o}^{3}$ must represent a volume. So, in Equation (36), it is easy to see that a thermal volume, $V_{T o}=\lambda_{o}^{3}$, has to be introduced. We must deal with the volume, since the transformation law of the length coincides with the one for the volume, since the factor, $\gamma^{-1}$, only applies in one direction, it is clear that we do not ask $\lambda_{o}^{3}$ to transform as $\lambda^{3}=\gamma^{-3} \lambda_{o}^{3}$, but as $\lambda^{3}=\gamma^{-1} \lambda_{o}^{3}$; that is, the wavelength is just transformed in one direction. Therefore, $V_{T}=\gamma^{-1} V_{T o}$; that is:

$$
\frac{P V_{o}}{N k T_{o}}=\sum_{l=1} a_{o l}\left[\frac{V_{T o}}{\frac{V_{o}}{N}}\right]^{l-1}
$$

Therefore, considering that $V_{T o}$ transforms as Equation (4), it is just necessary to prove that $a_{o l}$ are invariant. By analyzing their origin, we can relate the $a_{o l}$ coefficients with the Mayer cluster expansion coefficients, $b_{o l}[34]$. The following relationships are obtained:

$$
b_{o 1}=a_{o 1}, \quad b_{o 2}=a_{o 1} 2 b_{o 2}+a_{o 2} b_{o 1}^{2}, \quad b_{o 3}=a_{o 1} 3 b_{o 3}+a_{o 2} 4 b_{o 1} b_{o 2}+a_{o 3} b_{o 1}^{3}, \quad \text { etc. }
$$

A quick inspection shows that if we want the virial coefficients, $a_{o l}$, to be invariant, it is necessary that the Mayer coefficients, $b_{o l}$, are also invariant. For example, $b_{o 2}$ is calculated by the following relationship [34]:

$$
b_{o 2}=-\frac{2 \pi}{V_{T o}} \int_{0}^{\infty}\left(\exp \left[-\frac{U_{o}(\vec{r})}{k T_{o}}\right]-1\right) r^{2} d r
$$

Clearly, the potential must transform as the energy does, and this makes the exponential term an invariant. The integration contains a volume-like term divided by a thermal volume, which leads to an invariant. The same applies for other coefficients. So, we can conclude that the coefficients, $a_{o l}$, are invariants and the subscript "o" may be eliminated from the Mayer and virial coefficients. Therefore, we can ensure that the invariant form of the equation of state is accomplished in its virial expansion; that is:

$$
\frac{P V_{o}}{N k T_{o}}=\sum_{l=1} a_{o l}\left[\frac{V_{T o}}{\frac{V_{o}}{N}}\right]^{l-1}=\sum_{l=1} a_{l}\left[\frac{V_{T}}{\frac{V}{N}}\right]^{l-1}=\frac{P V}{N k T}
$$

where the subscript, "o", is eliminated from the right side of the identity in order to indicate that the quantities are measured in the moving frame. However, in the first instance, the thermal length could be considered as a quantity that does not transform as a length. Indeed, if we consider the definition of thermal wavelength:

$$
\lambda_{o}=\sqrt{\frac{2 \pi \hbar}{m k T_{o}}}
$$

At first instance, it can be thought that the wavelength transforms as:

$$
\lambda=\gamma^{1 / 2} \lambda_{o}
$$

Nevertheless, if we analyze the thermal length origin, by applying the Statistical Mechanics developed by Ares de Parga and López-Carrera [5] within the Nakamura formalism, we can assure that Equation (43) 
is not valid. Indeed, it has been demonstrated that the partition function is an invariant [5]. In the demonstration of this property, the following expression has to be considered:

$$
Z(T, V, N)=\frac{V^{N}}{N ! \hbar^{3 N}} \int d p^{3 N} \exp [-\beta H]
$$

The integral can be calculated in the rest frame, leading to:

$$
Z(T, V, N)=\frac{V^{N}}{N ! \hbar^{3 N}} \gamma^{N} \int d p_{o}^{3 N} \exp \left[-\beta_{o} H\right]
$$

where the factor, $\gamma^{N}$, has been introduced in order to leave invariant the phase space, as it has been demonstrated [5]. Since the thermal wavelength is defined from the fugacity $z=\lambda_{o}^{3} / v($ with $v=V / N)$, it is clear that it has to transform as a volume. This can be seen from the fact that $\lambda_{o}^{3}$ is proportional to $\int d p_{o}^{3} \exp \left[-\beta_{o} H\right]$, and a factor, $\gamma$, appears when the thermal wavelength is considered in the moving frame, as happens in a similar form in Equation (45). Therefore, the integration will lead us to define the thermal wavelength as:

$$
\lambda=\gamma^{-1} \lambda_{o}=\gamma^{-1} \sqrt{\frac{2 \pi \hbar}{m k T_{o}}}=\gamma^{-1} \sqrt{\frac{2 \pi \hbar q}{m^{2} c^{2}}}
$$

where $m$ represents the rest mass of the particles. Finally, since $u$ is an invariant, it can be concluded that the thermal wavelength transforms as a length, and the virial expansion is correct if the thermal wavelength is considered, as in Equation (46).

This method has been used to deal with relativistic systems for which a non-relativistic treatment will fail. Indeed, some examples are the pion gas virial expansion [35] and the calculation of the electric screening mass in hot hadronic matter [36], among others. If a simple inspection of the method is done, it is found that a thermal wavelength is also used (see Equation (1) of Dobado and Peláez [35]). In both cases, the coefficients are calculated to the second order, and consequently, the results meet the relativistic criterion if Equation (46) is considered when the thermal wavelength is used.

\section{Ideal Fermi and Bose Gases and the Blackbody}

\subsection{Ideal Fermi and Bose Gases}

The equation of state for the ideal Fermi and Bose gases are deduced by calculating the logarithm of the grand partition function [37]. This grand partition function, as is well-known, is defined by means of the fugacity and the partition function, both invariants. Therefore, the grand partition function and its logarithm are also invariants. As a consequence, the equation of state meets our criterion in both cases. Effectively, the obtained complete equations of state are:

Ideal Fermi Gas

$$
\left\{\begin{array}{c}
\frac{P}{k T}=\frac{1}{\lambda^{3}} f_{5 / 2}(z) \\
\frac{1}{V / N}=\frac{1}{\lambda^{3}} f_{3 / 2}(z) \\
\frac{\xi}{V}=\frac{3}{2} \frac{k T}{\lambda^{3}} f_{5 / 2}(z)
\end{array}\right.
$$

and 
Ideal Bose Gas

$$
\left\{\begin{array}{c}
\frac{P}{k T}=\frac{1}{\lambda^{3}} g_{5 / 2}(z)-\frac{1}{V} \log (1-z) \\
\frac{1}{V / N}=\frac{1}{\lambda^{3}} g_{3 / 2}(z)+\frac{1}{V} \frac{z}{1-z} \\
\frac{\xi}{V}=\frac{3}{2} \frac{k T}{\lambda^{3}} g_{5 / 2}(z)
\end{array}\right.
$$

where the functions, $f$ and $g$ are given by:

$$
\begin{aligned}
& f_{5 / 2}(z)=\sum_{l=1}^{\infty} \frac{(-1)^{l+1} z^{l}}{l^{5 / 2}}, \quad f_{3 / 2}(z)=\sum_{l=1}^{\infty} \frac{(-1)^{l+1} z^{l}}{l^{3 / 2}} \\
& g_{5 / 2}(z)=\sum_{l=1}^{\infty} \frac{z^{l}}{l^{5 / 2}} \quad g_{3 / 2}(z)=\sum_{l=1}^{\infty} \frac{z^{l}}{l^{3 / 2}}
\end{aligned}
$$

It has to be pointed out that Equations (47) and (48) can be deduced just by following, in the moving frame, the standard method used in the rest frame or simply by applying the relativistic transformation laws of the Planck-Einstein case [5]. It should be remembered that the redefined energy $\left(\xi=E-\frac{\mathbf{u}}{c} \cdot \mathbf{M}\right)$ and the thermal wavelength $(\lambda)$ transform as $\gamma^{-1} \xi_{o}=\gamma^{-1} E_{o}$ (see Equation (5)) and $\lambda=\gamma^{-1} \lambda_{o}$ (see Equation (46)). Normally, the complete equations of state are usually expressed in the rest frame of the gas; however, as is shown in Equations (47) and (48), they can be generalized to expressions in the moving frame satisfying our criterion. However, there is always a way to present the equation of the energy as a function of the thermodynamical quantities; that is, from Equation (47), we obtain for ideal Fermi gas:

$$
\xi=\frac{3}{2} P V
$$

which is obviously an invariant expression. For the Bose gas, using Equation (48), we do not obtain Equation (50). However, since $\frac{1}{V} \log (1-z)$ can be neglected [37], Equation (50) is also satisfied.

\subsection{The Blackbody}

It is a well-known result that the equation of state of the blackbody in its rest frame is:

$$
P V_{o}=\frac{1}{3} E_{o}
$$

which can be generalized in the moving frame, following our criterion, to:

$$
P V=\frac{1}{3} \xi
$$

When the energy density $\varsigma_{o}=\frac{E_{o}}{V_{o}}$ is calculated from basic principles, the result is:

$$
\varsigma_{o}=\frac{4}{c} \sigma T_{o}^{4}
$$

where $\sigma=\frac{\pi^{2} k^{4}}{15 \hbar^{3} c^{3}}$. If the density. $\rho=\frac{E}{V}$ is calculated in the moving frame [27,38], by using the Unruh-DeWitt excitation rate, the result does not coincide with the expected result; that is:

$$
\rho=\frac{4}{c} \sigma T_{o}^{4} \gamma^{2}\left(1+\frac{u^{2}}{3 c^{2}}\right)
$$


This is one of the reasons [38] why Landsberg and Matsas claimed the impossibility of a relativistic transformation law of temperature [39]. However, if the energy is redefined as expressed in Equation (5), $\xi=E-\frac{\mathbf{u}}{c} \cdot M=\gamma^{-1} \xi_{o}=\gamma^{-1} E_{o}$, and the redefined energy density is considered. $\varsigma=\frac{\xi}{V}$, it is obtained that:

$$
\varsigma=\frac{4}{c} \sigma \gamma^{4} T^{4}
$$

which coincides with Equation (53). since the redefined energy represents an invariant, $\varsigma=\varsigma_{o}$. In both cases, the rest and the moving frame, the equation of state is satisfied, Equations (51) or (52), and the invariance of the equation of state is accomplished; that is:

$$
P V=\frac{1}{3} \xi \Rightarrow P \gamma^{-1} V_{o}=\frac{1}{3} \gamma^{-1} E_{o} \Rightarrow P V_{o}=\frac{1}{3} E_{o}
$$

Therefore, the criterion is also valid in this case. Moreover, the fact that in Equation (55) a $\gamma$ factor appears does not represent an inconvenience. since it is deduced from fundamental principles [27,38].

\subsection{Summary}

In order to make a summary of the last results, let us notice that a further analysis of Equation (47) leads to:

$$
\frac{P V}{N k T}=\frac{f_{5 / 2}(z)}{f_{3 / 2}(z)}
$$

which is obviously an invariant equation. Nevertheless, this last expression does not completely describe the system. As in the case of the ideal gas, $P V=N k T$, we need the equation of the energy or the heat capacities in order to know all the thermodynamical properties of the system. For non-perfect gases, the heat capacities are given in the rest frame. and the coefficients must be corrected and will depend on $\gamma$. Therefore, we can conclude that a system will be described by an invariant equation of state, as can be seen in Equation (11) for the ideal gas, Equation (52) for the black-body and Equation (57) for the ideal Fermi gas, and an expression for the energy that can be expressed in the rest frame, but that can be directly generalized to the moving frame, as, for example Equations (29) and (30) for the non-perfect gas, or can be deduced from fundamental principles, such as Equation (55) for the blackbody and Equation (47) for the ideal Fermi gas.

\section{Equation of State for Nuclear Matter}

At the end of the last century, Shen et al. [40] constructed an equation of state for nuclear matter using the Relativistic Mean Field Theory. The aim was to perform calculations on neutron stars and supernova simulations. In this case, the free energy density in the rest frame is defined as

$$
f_{o}=\frac{E_{o}-T_{o} S}{a_{o}^{3}}
$$

where $E_{o}, S$ and $a_{o}$ represent the internal energy, entropy and the lattice constant, $a_{o}$ as the cube root of cell volume, $V_{o-\text { cell }}=a_{o}^{3}$ (the Wigner-Seitz cell [41]), in the rest frame, respectively. Then, the pressure can be calculated by using:

$$
P=\left.n_{B o}^{2} \frac{\partial\left[\frac{f_{o}}{n_{B o}}\right]}{\partial n_{B o}}\right|_{T_{o}, Y_{P}}
$$


where $n_{B_{o}}$ represent the baryon number density and $Y_{P}$ is the proton fraction, that is, the ratio of the number of protons to the number of baryons. It has to be asked if these last equations, Equations (58) and (59), satisfy the relativistic criterion. Analyzing Equation (58), it has to be noticed that it is necessary to substitute in the rest frame expression the value of $E_{o}$ by $\xi$, the redefined energy [23], so that the free energy density transforms as an invariant, that is:

$$
\xi=E-\frac{\mathbf{u}}{c} \cdot \mathbf{M}
$$

Therefore, the general expression of the free energy density is:

$$
f=\frac{\xi-T S}{a^{3}}=\frac{\gamma^{-1} E_{o}-\gamma^{-1} T_{o} S}{\gamma^{-1} a_{o}^{3}}=\frac{E_{o}-T_{o} S}{a_{o}^{3}}=f_{o}
$$

which is an invariant. It has to be noticed that $a$ is transformed like $a^{3}=\gamma^{-1} a_{o}^{3}$, as is the case of any length. Next, if the equation of state, Equation (59), is analyzed, it is obtained that:

$$
P=\left.n_{B o}^{2} \frac{\partial\left[\frac{f_{o}}{n_{B o}}\right]}{\partial n_{B o}}\right|_{T_{o}, Y_{P}}=\left.\gamma^{2} n_{B o}^{2} \frac{\partial\left[\frac{f}{n_{B o}}\right]}{\gamma^{2} \partial n_{B o}}\right|_{T_{o}, Y_{P_{o}}}=\left.n_{B}^{2} \frac{\partial\left[\frac{f}{n_{B}}\right]}{\partial n_{B}}\right|_{T, Y_{P}}
$$

Equation (62) preserves the same form in any frame, that is, the invariance of the form. In this case, the Mean Field Theory generates an equation of state, which is consistent with the relativistic criterion.

\section{The Rubber Band}

At the moment, we have analyzed equations of state that can be expressed in a invariant form when the systems are observed from a relativistic moving frame by using a proposal for the relativistic transformation laws of thermodynamical quantities. However, as a referee suggested to us, it will be interesting to study an equation of state that fulfills all consistency relations from standard thermodynamics in the rest frame, but which would be ruled out only due to the newly derived constraints from relativistic thermodynamics. At first instance, the rubber band may represent such a case. However, a forward analysis shows that the criterion permits us to know how the rubber band equation has to be generalized for relativistic reference frames.

Indeed, the rubber band can be considered as a simple thermodynamical system [28]. Even if it just represents an illustrative example, since a better analysis must be done by modeling the polymer elasticity from a statistical method, it is an illustrative example of a system that satisfies all the thermodynamical requirements. Indeed, a Carnot cycle can be constructed, and all the normal properties derived from it are obtained. Its entropy in the rest frame, $S_{o}$, is:

$$
S_{o}=S_{o e}+c_{o} L_{o e} \ln \frac{U_{o}}{U_{o e}}-\frac{b_{o}}{2\left(L_{o 1}-L_{o e}\right)}\left(L_{o}-L_{o e}\right)^{2}
$$

where $b_{o}$ and $c_{o}$ represent phenomenological constants, $S_{o e}$ is the unstretched rubber entropy, $L_{o}, L_{o e}$ and $L_{o 1}$ are the length, the unstretched length and the elastic limit length, respectively, and $U_{o}$ is the internal energy (the subscript, " $O$ " designs the quantities measured in the rest frame and the subscript, " $e$ " denotes the unstretched quantities):

$$
U_{o}=c_{o} L_{o e} T_{o}
$$


It is observed that it is not possible to express the entropy as an invariant and, at the same time, to have an internal energy that transforms as an energy unless the constants, $c_{o}$ and $b_{o}$ are corrected in the relativistic moving frame; that is, in the moving frame, the entropy must be expressed as:

$$
S=S_{e}+c L_{e} \ln \frac{U}{U_{e}}-\frac{b}{2\left(L_{1}-L_{e}\right)}\left(L-L_{e}\right)^{2}
$$

and:

$$
U=c L_{e} T
$$

In order that $U$ transforms as an energy and considering that $L_{e}=\gamma^{-1} L_{o e}$ and $T_{e}=\gamma^{-1} T_{e}$, following the relativistic transformations, $c$ must transforms as $c=\gamma c_{o}$. A similar reasoning leads us to $b=\gamma b_{o}$. Therefore, the criterion gives us a technique to correct the empirical constants, in order to express the entropy in the relativistic moving frame as an invariant. A similar situation has been presented above in Section 4 , where the virial coefficients were analyzed.

\section{Concluding Remarks}

It has been shown that there is an acceptable criterion to test if an equation of state is physically acceptable for relativistic reference frames by using the relativistic transformation laws of thermodynamics. The criterion has been used in order to verify the consistency of several equations of state, relativistic or not, as the relativistic ideal gas, the ideal Fermi and Bose gases, the blackbody, the equation of state for nuclear matter and the non-relativistic (low temperatures) van der Waals gas. In the case of an empirical equation of state, it is shown how the coefficients must be defined in the moving frame in order to satisfy the relativistic criterion. An analysis has been done for the equations, which make a complete description of the system and which are normally expressed in the rest frame and can be generalized to the moving frame. When an equation of state is obtained by a statistical method, as the virial expansion, the relativistic approach provides a method of analysis for the significance and correction of each of the coefficients. It has been shown that the virial expansion is valid in a relativistic reference frame if the thermal wavelength is corrected. Finally, this relativistic criterion must be taken into account at the moment of considering an equation of state coming from the empirical or from a statistical model when it is analyzed from a relativistic moving frame.

\section{Acknowledgments}

This work was partially supported by C.O.F.A.A and E.D.I., I.P.N.

\section{References}

1. Tykodi, R.J.; Hummel, E.P. On the equation of state for gases. Am. J. Phys. 1973, 41, 340-343.

2. Angulo-Brown, F.; Olivar-Romero, F. About the thermodynamical self-consistency of some equations of state 2012. MSc.Thesis, ESFM IPN, Mexico City, Mexico, 2012.

3. Carathéodory, C. Untersuchungen ber die Grundlagen der Thermodynamik. Math. Ann. 2012, 67, 355-386. 
4. Frankel, T. The Geometry of Physics: An Introduction; Cambridge University Press: Cambridge, UK, 2003; Chapter 6, p. 181.

5. Ares de Parga, G.; López-Carrera, B. Relativistic statistical mechanics vs. relativistic thermodynamics. Entropy 2011, 13, 1664-1693.

6. Planck, M. Zur dynamik bewegter systeme. Ann. Phys. 1908, 331, 1-34.

7. Einstein, A. Über das Relativitätsprinzip und die aus demselben gezogenen Folgerungen. Jahrbuch der Radioaktivität und Elektronik 1907, 4, 411-462.

8. Landsberg, P.T.; Matsas, G.E. Laying the ghost of the relativistic temperature transformation. Phys. Lett. A 1996, 223, 401-403.

9. Ott, H. Lorentz-transformtion der warme und der temperatur. Z. Phys. 1963, 175, 70-104.

10. Landsberg, P.T. Does a moving body appear cool? Nature 1966, 212, 571-572.

11. Rohrlich, F. True and apparent transformations, classical electrons, and relativistic thermodynamics. Il Nuovo Cimento B 1966, XLV, 6200-6207.

12. Van Kampen, N.G. Relativistic thermodynamics of moving systems. Phys. Rev. 1968, 173, 295-301.

13. Israel, W. Nonstationary irreversible thermodynamics: A causal relativistic theory. Ann. Phys. 1976, 100, 310-331.

14. Israel, W. Thermodynamics of relativistic systems. Physica A 1981, 106, 204-214.

15. Balescu, R. Relativistic statistical thermodynamics. Physica 1968, 40, 309-338.

16. Staruszkiewicz, A. Relativistic transformation laws for thermodynamical variables with applications to classical electron theory. Il Nuovo Cimento A 1966, XLV, 6632-6636.

17. Nakamura, T.K. Three views of a secret in relativistic thermodynamics. Prog. Theor. Phys. 2012, 128, 463-475.

18. Möller, C. The theory of Relativity; International Series of Monographs on Physics; Clarendon Press: Oxford, UK, 1972; Chapter 7.

19. Requardt, M. Thermodynamics meets special relativity-Or what is real in physics? arXiv 2008, 08012639, 2639:1-2639:27.

20. Przanowski, M.; Tosiek, J. Notes on thermodynamics in special relativity. Phys. Scripta 2011, 84, doi:10.1088/0031-8949/84/05/055008.

21. Jüttner, F. Das Maxwellsche Gesetz der Geschwindigkeitsverteilung in der Relativtheorie. Ann. Phys. 1911, 339, 856-882.

22. Cubero, D.; Casado-Pascual, J.; Dunkel, J.; Talkner, P.; Hänggi, P. Thermal equilibrium and statistical thermometers in special relativity. Phys. Rev. Lett. 2007, 99, 170601:1-170601:4.

23. Ares de Parga, G.; López-Carrera, B. Redefined relativistic thermodynamics based on the Nakamura formalism. Physica A 2009, 388, 4345-4356.

24. Nakamura, T.K. Relativistic energy-momentum of a body with a finite volume. Space Sci. Rev. 2006, 122, 271-278.

25. Dunkel, J.; Hänggi, P.; Hilbert, S. Non-local observables and lightcone-averaging in relativistic thermodynamics. Nat. Phys. 2009, 5, 741-747.

26. Greiner, W.; Neise, L.; Stcker, H. Thermodynamics and Statistical Mechanics; Springer-Verlag: New York, NY, USA, 1995; Chapter 8. 
27. Ares de Parga, G.; López-Carrera, B.; Angulo-Brown, F. A proposal for relativistic transformations in thermodynamics. J. Phys. A Math. Gen. 2005, 38, 2821-2834.

28. Callen, H.B. Thermodynamics and an Introduction to Thermostatistics; John Wiley \& Sons: Hoboken, NJ, USA, 1985; Chapters 1 and 2, pp. 28, 37.

29. Currie, D.G.; Jordan, T.F.; Sudarshan, E.C.G. Relativistic invariance and hamiltonian theories of interacting particles. Rev. Mod. Phys. 1963, 35, 350-375.

30. Pryce, M.H.L. The mass-centre in the restricted theory of relativity and its connexion with the quantum theory of elementary particles. Proc. R. Soc. Lond. A 1948, 195, 62-81.

31. Thomas, L.H. The relativistic dynamics of a system of particles interacting at a distance. Phys. Rev. 1952, 85, 868-872.

32. Bakamjian, B.; Thomas, L.H. Relativistic particle dynamics. II. Phys. Rev. 1953, 92, 1300-1310.

33. Gill, T.; Zachary, W. Two mathematically equivalent versions of maxwells equations. Found. Phys. 2011, 41, 99-128.

34. Reference [26], a comparison between last expression in p 403, Equation (16.3) of p 404, the van der Waals equation and the virial expansion, Equation (16.61) of p 414, shows that the van der Waals equation is a virial expansion to second order.

35. Dobado, A.; Peláez, J.R. Chiral symmetry and the pion gas virial expansion. Phys. Rev. D 1998, 59, 034004:1-034004:9.

36. Eletsky, V.L.; Kapusta, J.I.; Venugopalan, R. Screening mass from chiral perturbation theory, virial expansion, and the lattice. Phys. Rev. D 1993, 48, 4398-4407.

37. Huang, K. Statistical Mechanics; John Wiley \& Sons: Hoboken, NJ, USA, 1963; Chapters 9 and 12, pp. 200-201, 266-267.

38. López-Carrera, B.; Antonio, R.M.; Ares de Parga, G. The 2.7 K black-body radiation background reference frame. Chin. Phys. B 2010, 19, 040203:1-040203:5.

39. Landsberg, P.T; Matsas, G.E.A. The impossibility of a universal relativistic temperature transformation. Phys. A: Stat. Mech. Appl. 2004, 340, 92-94.

40. Shen, H.; Toki, H.; Oyamatsu, K.; Sumiyoshi, K. Relativistic equation of state of nuclear matter for supernova and neutron star. Nucl. Phys. A 1998, 637, 435-450.

41. Wigner, E.; Seitz, F. On the constitution of metallic sodium. Phys. Rev. 1933, 43, 804-810.

(c) 2013 by the authors; licensee MDPI, Basel, Switzerland. This article is an open access article distributed under the terms and conditions of the Creative Commons Attribution license (http://creativecommons.org/licenses/by/3.0/). 